\title{
Closing the gap between nano- and pore scale: upscaling strategies using computational geometry
}

\author{
RICARDA D. ROHLFS ${ }^{1 *}$, INNA KURGANSKAYA ${ }^{1}$, \\ CORNELIUS FISCHER ${ }^{2}$ AND ANDREAS LUTTGE ${ }^{1}$
}

${ }^{1}$ MARUM \& FB05, Universität Bremen, 28359 Bremen,

Germany (*correspondence: riccy@uni-bremen.de)

${ }^{2}$ Institut für Ressourcenökologie, Abteilung Reaktiver

Transport (FS Leipzig), Helmholtz-Zentrum Dresden-

Rossendorf, 04318 Leipzig, Germany

Geochemists nowadays have access to a variety of methods for modeling fluid-solid interactions at almost all accessible scales. At the smallest molecular level, Quantum Mechanical methods allow us to understand reaction pathways. These can be upscaled with Kinetic Monte Carlo simulations for atomistic systems. When simulating crystal dissolution, the challenging part is to further upscale KMC modelling results to pore scale and reservoir scale where Reactive Transport methods can be applied.

To represent the chemical complexity of reactive mineralfluid systems at a larger scale, the system has to be simplified while retaining important key parameters. Our algorithmic approach is based on computational geometry. We use nonEuclidean distance functions to characterize the influence of atomic step generators, e.g., screw dislocations, on the development of surface morphology during dissolution [1]. We have further expanded this approach to the calcite lattice, parametrized with KMC simulations [2], and now present strategies to bridge the gap between KMC scale and larger systems. Calcite is a ubiquitous mineral in sedimentary rocks and is an ideal candidate for our methodological tests.

Since this method is inherently scale-unlimited its results can be verified by comparison with reactive surface topographies studied experimentally, e.g., with Atomic Force Microscopy (nm- $\mu \mathrm{m}$ range) and Vertical Scanning Interferometry (nm-mm range). We investigate the mechanistic connection between surface geometry and associated rate variance. From a systematic statistical analysis of morphological features, we derive upscaling rules suitable for the problem of connecting the atomistic scale to pore scale processes.

[1] Rohlfs et al., Minerals 2018, 8, 133. [2] Kurganskaya and Luttge, J. Phys. Chem. C 2016, 120, 12, 6482-6492. 